Cahiers d'études africaines

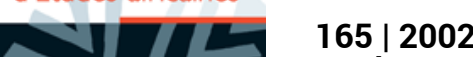

Varia

\title{
Territorialisation du savoir et invention de la Méditerranée*
}

Jean Schmitz

\section{OpenEdition}

1 Journals

Édition électronique

URL : http://journals.openedition.org/etudesafricaines/139

DOI : 10.4000/etudesafricaines.139

ISSN : 1777-5353

Éditeur

Éditions de l'EHESS

\section{Édition imprimée}

Date de publication : 1 janvier 2002

Pagination : 143-160

ISBN : 978-2-7132-1420-2

ISSN : 0008-0055

\section{Référence électronique}

Jean Schmitz, "Territorialisation du savoir et invention de la Méditerranée* », Cahiers d'études africaines [En ligne], 165 | 2002, mis en ligne le 25 mai 2005, consulté le 03 février 2021. URL : http:// journals.openedition.org/etudesafricaines/139; DOI : https://doi.org/10.4000/etudesafricaines.139 


\section{Jean Schmitz}

\section{Territorialisation du savoir et invention de la Méditerranée*}

Deux livres collectifs regroupant quelquefois les mêmes participants viennent de prendre pour objet les représentations de l'espace méditerranéen au $\mathrm{XIX}^{\mathrm{e}}$ siècle. Le premier ouvrage rassemble du côté français les contributions à un séminaire tenu à 1'Université Paris-VII-Denis Diderot dans les années 1992-1994 ${ }^{1}$ tandis que le second regroupe les contributions à un colloque international tenu à Athènes en 1995 (les 8-10 juin) rejoignant des recherches menées par l'Institut de recherches néohelléniques. C'est la construction scientifique de l'espace méditerranéen au cours des expéditions scientifiques et militaires, envoyées par la France entre la fin du XVIII ${ }^{\mathrm{e}}$ et la moitié du $\mathrm{XIX}^{\mathrm{e}}$ siècle, qui sert de trait d'union aux préoccupations scientifiques tant du côté de la Grèce qu'en France : expédition d'Égypte à l'époque du Directoire (1798-1801), en Morée (actuel Péloponnèse) à la fin du règne de Charles X (1829-1831), en Algérie enfin sous la monarchie de Juillet (18391842). Ce sont souvent les mêmes hommes ou leurs disciples qui participèrent aux deux dernières expéditions.

Ces expéditions militaro-scientifiques, «dispositifs» originaux de savoir-pouvoir au sens de Michel Foucault (Bourguet a), sont une occasion pour les auteurs rassemblés, quelquefois à deux reprises, de traverser en tout sens des corpus de textes de taille souvent considérable. La Description de l'Égypte, texte publié entre 1809 et 1828, compte 10 volumes d'atlas et 9 de textes. La Commission scientifique de Morée est de dimension plus modeste (4 vols) alors que la Commission d'exploration scientifique de

* À propos de : a) Bourguet, M.-N., Lepetit, B., Nordman, D. \& Sinarellis, M. (dir.), L'invention scientifique de la Méditerranée. Égypte, Morée, Algérie, Paris, Éditions de l'EHESS («Recherches d'histoire et de sciences sociales »), 1998; b) Bourguet, M.-N., Nordman, D., Panayotopoulos, V. \& Sinarellis, M. (dir.), Enquêtes en Méditerranée. Les expéditions françaises d'Égypte, de Morée et d'Algérie, Athènes, Institut de recherches néohelléniques/FNRS, 1999.

Le renvoi, dans le texte, aux contributions figurant dans l'un des deux ouvrages est indiqué par la lettre a (1998) ou b (1999).

1. Ce programme était conduit en collaboration par Marie-Noëlle Bourguet, Bernard Lepetit, Daniel Nordman et Maroula Sinarellis. 
l'Algérie comprend 37 volumes publiés beaucoup plus tard. Elles mettent en rapport la construction des savoirs naturalistes et l'entreprise impériale autour du rapport privilégié à l'espace.

Pour l'anthropologue ou l'africaniste, ces expéditions représentent un double intérêt. Le modèle de l'expédition militaro-scientifique fut transféré au sud du Sahara comme en témoigne l'exploration organisée par la Commission de la carte de la Sénégambie, le «bureau arabe » créé par Faidherbe dans les années 1857-1861: ce dernier avait été officier du génie en Algérie dans les années 1840. L'héritage de ces expéditions sera important. Récemment, J.-L. Amselle (1996) voit dans les expéditions d'Égypte, d'Algérie et de Sénégambie, un des fondements du multiculturalisme à la française.

En second lieu la perspective adoptée soulignant la complexité des rapports entre science et empire est l'occasion d'observer la mise en œuvre de pratiques comme celle du terrain des géologues ou des archéologues ou de notions comme celle de région en géographie, de système en géologie, de modèle ou de type idéal au sein des beaux-arts qui essaimeront plus tard vers les sciences sociales. Dans un article célèbre, J. Berque (1956) souligna à quel point la connaissance du Maghreb, sur laquelle allait s'édifier une partie de la sociologie durkheimienne, fut redevable d'un certain nombre de concepts clés à l'exploration scientifique de l'Algérie.

Or la construction de ces pratiques ou de ces catégories fut un élément essentiel d'un vaste mouvement de «territorialisation du savoir » (Bourguet a) ou de la connaissance (Lepetit a) qui s'opère à la charnière des XVIII ${ }^{\mathrm{e}}$ et $\mathrm{XIX}^{\mathrm{e}}$ siècles. Dynamique scientifique à laquelle ces trois expéditions offrent des champs d'expérimentation dispersés sur les deux rives de la Méditerranée qu'elles contribuent à unifier. On peut alors s'interroger sur les rapports entre l'objectivisme durkheimien et ce primat de l'espace qui l'a largement précédé et qu'il faut concevoir aussi bien en termes de détermination par les «conditions naturelles» qu'au niveau des techniques d'observation: observation directe, mesures et relevés sur place.

En cette fin de siècle, par un curieux retour de l'histoire, l'anthropologie et les cultural studies anglo-saxonnes héritières du linguistic turn (J. Clifford, A. Appadurai, R. Robertson...) invoquent la «déterritorialisation» de la culture ou la «culture voyageuse », célébrant à nouveau le cosmopolitisme, déjà à l'ordre du jour au XVIII ${ }^{\mathrm{e}}$ siècle. Bien que cette remise en cause porte également sur le substrat des cultures et sur les techniques d'observation, il serait anachronique d'y voir l'inversion pure et simple du premier processus.

Voyons comment, et sous l'effet de quelles recompositions, le mouvement premier - la territorialisation du savoir — s'est effectué à la charnière des $\mathrm{XVIII}^{\mathrm{e}}$ et XIX ${ }^{\mathrm{e}}$ siècles.

En prélude à cette reconfiguration on assiste, à la fin du XVIII ${ }^{\mathrm{e}}$ siècle, à la modification de la nature des collections et à celle des voyages, qui toutes deux avaient pour but d'offrir un contrepoint aux textes. 


\section{Modification de la pratique du voyage}

$\mathrm{Au}$ milieu du XVIII ${ }^{\mathrm{e}}$ siècle intervient une triple modification des objets de science - la finalité des voyages —, des modalités de l'observation et de l'écriture du voyage.

Alors qu'auparavant les objets singuliers venaient enrichir un cabinet de curiosités, ce sont dorénavant les objets ordinaires ou les régularités qui sont collectés. Parallèlement ce ne sont plus seulement les «contrées sauvages » qui attirent les voyageurs, mais les pays proches comme la Méditerranée pour Rousseau (Bourguet a : 5). B. Lepetit (a : 110) oppose les explorations maritimes caractéristiques du $\mathrm{XVIII}^{\mathrm{e}}$ siècle qui se déploient linéairement et les explorations continentales du XIX ${ }^{\mathrm{e}}$ siècle, liées parfois à la conquête, qui raisonnent en termes de semis, de quadrillages et de surfaces.

Se pose alors la question des modalités de passage de l'observation linéaire ou ponctuelle à l'idéal de la préhension d'une configuration générale par l'observation indirecte ou les modalités de la couverture raisonnée du terrain. Dans le fameux Voyage en Syrie et en Égypte (1787) de Volney, qui inspira Bonaparte, on assiste à une sorte de dédoublement du voyageur : l'impression directe est valorisée à condition d'être suivie d'opérations de décomposition et recomposition des faits selon les règles de la philosophie sensualiste. D'où l'usage de la comparaison. En ce qui concerne le paysage de la Méditerranée, plusieurs contributions citent le fameux passage où Bory de Saint-Vincent compare le Péloponnèse à la Provence et aux montagnes de Hyères. Les paysages fournissent des indices de leur appartenance à une aire plus vaste, indices qui ne procèdent plus directement de la vision du voyageur.

$\mathrm{Au}$ niveau de l'écriture même du paysage, s'opèrent des ruptures majeures. Pour le savant voyageur de la fin du XVIII ${ }^{\mathrm{e}}$ siècle, les observations ne peuvent être déconnectées de la perception sensible qui s'exprime dans les pratiques de contemplation de paysages. Plus tard durant les expéditions, Denon et Bory publient séparément la relation du récit du périple qu'ils ont effectué. Car pour Jomard, géographe de l'expédition d'Égypte, les voyageurs ont évité de se «mettre en scène », ce qui entraîne parallèlement la séparation entre la description du voyage, la « relation » où s'exprime des «opinions », et le «mémoire » où les faits sont établis, discutés et interprétés (Dias in a : 163). Cette évolution s'achève dans les années 1830 lorsque le récit de voyage du naturaliste ne joue plus qu'un rôle annexe dans des traités où la pensée scientifique apparaît comme le fruit d'une recomposition d'observations dont on ne saisit ni l'ordre ni le contexte spatio-temporel (Briffaud in a : 289-297).

\section{Modification de la pratique de la collection}

La collecte de nouveaux spécimens pour les collections du Jardin du roi était le souci permanent de son jardinier en chef, André Thouin. Or la Révolution et les guerres napoléoniennes vont modifier radicalement les modalités de collecte des spécimens (Spary in a). 
À la fin des années 1780 l'approvisionnement en nouveaux spécimens reposant sur un système d'obligations et de crédits obéissait à une économie du don qui attestait du statut du partenaire au sein d'un réseau de correspondants (ibid. : 121). À l'autre bout de la chaîne les transferts de plantes et les voyages qui les précédaient étaient justifiés par le bénéfice qu'on pouvait attendre du commerce qui en résulterait.

Après 1789 les sciences naturelles et les arts furent considérés comme des disciplines idéales à enseigner aux jeunes citoyens. Surtout, la quête des collections d'histoire naturelle et celle des objets d'art s'étendirent aux territoires conquis. La collecte dépendait dorénavant des rapports entre États et des victoires politiques (ibid. : 124). Thouin suit les armées aux PaysBas puis en Italie pour classer et recenser les spécimens tirés des collections confisquées, travail parallèle à celui qui fut effectué par Vivant Denon en matière d'objets d'art. Les objets affluaient vers le Muséum d'histoire naturelle et le Muséum national des arts (qui allait devenir le Louvre), puis la lumière et l'instruction étaient rediffusées jusqu'aux divers centres régionaux de la République.

\section{La territorialisation du savoir dans les sciences naturelles}

Le processus de territorialisation entamé dans les années 1760 suppose deux choses du point de vue des techniques et des pratiques. Le terrain, à savoir que la connaissance de l'objet observé ne peut se faire que sur place, après l'identification du site, même si la comparaison et l'identification se font au sein de l'espace de la collection, de spécimens réels ou de représentations, sous forme de planches gravées, de maquettes, etc.

Dès lors les techniques d'observation, combinant selon des rhétoriques de la preuve changeantes, mesures, récits, dessins, deviennent des lieux communs partagés par plusieurs corps de spécialistes parmi lesquels on compte les savants, mais pas exclusivement. Lieux communs qui peuvent servir de trait d'union (le dessin entre l'architecture et les mathématiques), faire l'objet de transfert - techniques des ingénieurs des mines, civils ou militaires, vers l'archéologie — ou enfin être l'enjeu d'affrontements entre corps professionnels ou disciplines naissantes : relevés de monument par des archéologues, des ingénieurs ou des architectes, cartographie de l'armée (brigade topographique) ou des ingénieurs géographes...

Revenons sur ces transferts ou ces concurrences dans les domaines de la cartographie et de l'archéologie à travers les deux premières expéditions, l'Algérie n'ayant pas donné lieu à des entreprises cartographiques importantes.

Bernard Lepetit (a : 105) s'attache particulièrement à la cartographie qui marque le recouvrement du champ des savants et de celui des militaires. En Égypte la carte est préparée conjointement par les ingénieurs (géographes, Ponts et Chaussées) de la commission et des officiers du génie 
appartenant à l'armée. Les relevés à la planchette fondés sur un travail de triangulation topographique sont cantonnés à une bande étroite le long du Nil et sur la côte: Alexandrie, Le Caire... C'est aussi en Égypte qu'on assiste à la participation enthousiaste des ingénieurs des Ponts et Chaussées à la découverte archéologique de l'Égypte.

La cartographie détaillée de la Morée et de la Grèce fut l'application des études mathématiques, topographiques et d'arpentage mises au point à la fin du $X I_{I I}{ }^{\mathrm{e}}$ siècle : arpentage et triangulation (Saïtas in b : 105-129). Quatre membres de l'expédition scientifique, géographes et ingénieurs, dont Puillon de Boblaye et Bory de Saint-Vincent, collaborèrent avec la brigade topographique de l'armée française (ibid. : 107) pour lever les trois péninsules du Péloponnèse. Sont importées en archéologie des pratiques de terrain qui relèvent d'un apprentissage d'ingénieur et non d'une archéologie de cabinet (Lepetit a : 113) suivant un certain nombre d'instructions énumérant diverses opérations: journal de fouilles avec boussole et montre, carte, relevé exact du matériel, quadrillage du site, sondages, fouilles en ligne.

Cette territorialisation ne s'est pas faite selon les mêmes modalités au sein de ce qui allait devenir des disciplines lors de leur inscription dans le système universitaire. Commençons par la géographie, plaque tournante de tous ces savoirs, puis par les deux références fondatrices : la géologie née à la fin du XVIII ${ }^{\mathrm{e}}$ siècle et la médecine néo-hippocratique. Puis abordons la botanique et la zoologie et les beaux-arts.

Deux tensions parcourent cette territorialisation. La première opposerait d'un côté l'autopsie, le « terrain » naturaliste qui l'accompagne, et de l'autre les textes, récits de voyage ou catalogues de collection. Cette opposition est plus complexe que les controverses auxquelles on serait tenté d'attribuer les noms de Geoffroy Saint-Hilaire (qui participa à l'expédition d'Égypte) et de Cuvier en zoologie, d'Alexandre de Humboldt qui parcourut l'Amérique latine et de Pyramidus de Candolle en géographie botanique, ou bien de Blouet et de Bory de Saint-Vincent en matière d'archéologie et beauxarts qui tous deux participèrent à l'expédition de Morée (voir infra). On verra que ces deux styles de science s'interpénètrent plus qu'ils ne s'opposent rigoureusement. On essaiera de faire le parallèle avec les débats opposant architectes ou spécialistes des beaux-arts.

Examinons tout d'abord les deux expériences contrastées de l'Égypte et de l'Algérie en ce qui concerne la géographie.

Jomard (1777-1862), ingénieur géographe de l'expédition d'Égypte, chef du département des cartes géographiques de la bibliothèque royale (Forgeau in a : 40), affirme le privilège de la chose vue par rapport au on-dit, ou la supériorité des savants sur les antiques. Il élabore toute une technique de relevé de terrain : dans la Description chaque site est déterminé par sa longitude et sa latitude, ainsi que par sa position par rapport au Nil (ibid. : 39), et chaque élément des monuments est mesuré. L'importance de cette mise en site grâce à la topographie est inscrite en creux dans le non-déchiffrement des hiéroglyphes, obligeant à importer les méthodes des paléontologues en 
archéologie plutôt qu'à analyser un corpus, ce qui ne sera possible qu'après la découverte de Champollion. Car, selon l'expression de Jomard, les monuments égyptiens étaient le « miroir» du pays qui les a vus naître, les savants se voulaient les déchiffreurs de la civilisation pharaonique (ibid. : 50). Jomard souligne les deux avantages de la carte. Elle permet une vision synthétique d'un seul coup d'œil qui répond à un principe d'économie et permet de « suppléer» l'accumulation des détails (Dias in a : 182). Second avantage, cette présentation synoptique donne l'illusion d'un regard d'en haut, de type panoptique, qu'on peut avoir dans le panorama (ibid. : 175).

À l'inverse de la victoire sans conteste du « terrain » que pourrait suggérer cette présentation des écrits de Jomard durant l'expédition d'Égypte, D. Nordman (a: 71-95) replace l'exploration de l'Algérie dans le débat coextensif à la géographie sur les mérites respectifs de l'observation personnelle, l'autopsie, ou du recours à l'autorité des textes. Il s'interroge tout d'abord sur le rapport entre le terrain et la mise en écriture ultime puisque, dans le cas de l'Algérie, le premier dura trois ans (1839-1842) alors que la rédaction de L'exploration scientifique s'effectua en vingt ans (18441867). Ce «terrain sans terrain » impose de trouver des substituts au contact direct - recueil de planches ou types humains regroupés dans une prison (Nordman a : 92).

Dans sa réflexion sur la notion de région, Nordman établit que ce « terrain sans terrain » est une notion coextensive de l'exploration elle-même. Si les descriptions de géographie physique de Renou et de Carette s'appuient sur la perception linéaire du voyageur, elles s'enrichissent de ce qui n'a pas été perçu. Arrêtons-nous sur Carette (1808-1890), officier saint-simonien arrivé en 1835 en Algérie qui prend part à des expéditions militaires et participe à la Commission en 1839, ayant beaucoup écrit sur la géographie de l'Algérie et en particulier sur la Kabylie (Études sur la Kabylie proprement dite, 1848). Dans les six processus de reconstruction intellectuelle de la géographie algérienne que Nordman (b) identifie chez les deux auteurs, il convient de noter la faible part de ceux qui font appel à la vision directe.

Les trois premiers s'appuient sur les témoignages indirects. Les lieux, ou «points principaux du canevas » qui permettent à Carette d'identifier les routes, sont localisés à partir de trois sortes de géographie, dont celle « testimoniale» qui s'appuie sur les indigènes perçus comme des «boussoles intelligentes » (ibid. : 149). Le second principe, celui de l'analogie, est utilisé par un voyageur naturaliste du Muséum. Le troisième schéma fait appel à la géologie : c'est le système qui n'est pas de l'ordre du visible. Renou confirme les systèmes montagneux transcontinentaux qu'a identifiés le géologue Élie de Beaumont, lequel avait déjà rapproché toutes les chaînes qui traversent l'Algérie des trois principaux axes de l'Europe méridionale.

Seuls les deux principes suivants de découpage reposent sur des descriptions que peuvent recueillir le voyageur. Il s'agit du partage zonal qui oppose, selon Carette, deux régions, le Tell, région du labourage et des 
moissons, et le Sahara, région des pasteurs et des jardiniers des oasis (Frémeaux 1996) et du partage en trois segments méridiens (les provinces de l'ouest, du centre et de l'est), divisés chacun en deux régions, Tell et Sahara, soit en tout six régions (p. 156).

Même le dernier principe — l'essai de division régionale — fait appel à des critères non géographiques. Dans son étude sur la Kabylie (1848) c'est le langage qui différencie Arabes et Kabyles, ou c'est le partage selon les tribus qui est antérieur et décisif, permettant le choix a posteriori d'une limite topographique parmi toutes les autres. Or Carette choisit celui de cantons, en référence à la subdivision administrative française...

En réalité ce mouvement de territorialisation provient moins de la géographie que de la géologie et de la médecine hippocratique dans les trois expéditions. Le fondement de la saisie globale du territoire est à chercher du côté de la géologie comme on va le voir pour la Morée et l'Algérie. Pour Puillon de Boblaye, qui fait partie de l'expédition de Morée, puis de la Commission d'Algérie (Nordman a : 76) ce sont les causes géognostiques, soit la nature minéralogique du sol, qui « donnent les principales conditions d'existence des peuples » (Sinarellis a : 133, 139). C'est là également qu'il faut chercher l'unité de la Méditerranée. M. Sinarellis essaie d'élucider comment Puillon de Boblaye et Théodore Virlet, qui ont écrit la partie Géologie et minéralogie de l'expédition de Morée, rapprochent l'histoire de l'Homme et l'histoire de la Terre. Elle souligne l'importance du relais d'Élie de Beaumont, catastrophiste comme Cuvier, auquel il succède en 1832, et qui supervise les recherches géologiques en Grèce et plus tard en Algérie. Puillon de Boblaye distingue plusieurs systèmes de montagne : l'Olympique, le système Pindique... Cela permet l'emboîtement des études régionales dans la géographie minéralogique globale, parallèle à celui qui lie l'histoire de la géologie actuelle et ancienne et permet de comprendre l'histoire de l'Homme dans une histoire en abîme qui fait que les Anciens, et en particulier Strabon, auraient entrevu la théorie des soulèvements (p. 138).

Le second fondement de ce primat de l'espace réside dans la doctrine de l'hippocratisme dont l'influence s'étend jusque dans les années 1830 : humorisme (la maladie se trouve dans les liquides du corps), nécessité de décrire les symptômes externes de la maladie, épidémiologie qui exigeait de cartographier les maladies en fonction de l'environnement (doctrine climatologique présente dans le traité hippocratique Des airs, des eaux et des lieux (Osborne a : 186)). En Égypte le climat fonde la raciologie. Le facteur climatique influe sur la forme du crâne et les différences raciales. Jomard compare la forme des crânes des Égyptiens (des cheikhs de villages) modernes et celle des Anciens, soit les momies de Thèbes, ce qui lui permet de dire qu'ils sont identiques et de leur dénier un caractère "éthiopien » pour leur attribuer un caractère arabe (p. 169).

Ce renouveau de l'hippocratisme est dû à deux personnages, le zoologiste Isidore Geoffroy Saint-Hilaire (p. 190) et Émile Littré, humaniste et philologue de 1839 à 1861 qui déshistoricisa Hippocrate en s'aidant des 
médecins en poste en Algérie, en particulier Perrier et son programme assimilationniste de 1830-1840.

La seconde tension qui parcout cette territorialisation concerne le rapport au temps et le régime d'historicité (Hartog 1995). Le primat de l'espace permettant d'établir la continuité entre l'histoire de la Terre et celle des Hommes, c'est la stratigraphie géologique et le catastrophisme - doctrine de Cuvier et de son disciple Élie de Beaumont, supplanté ultérieurement par l'évolutionnisme - qui semblent triompher. Par le biais de l'archéologie (architecture, beaux-arts), le primat du géologique entraîne la mise au temps des Anciens, ou du Moyen Âge des sociétés observées, leur mise hors temps s'effectuant à la fin du XIX ${ }^{\mathrm{e}}$ siècle (Fabian 1983). Or les apories de la géographie botanique, le décalage entre la distribution normale des phénomènes et celle qui est observée, nécessitèrent la référence à l'histoire et préfigurent l'évolutionnisme. Le même mouvement peut s'observer en zoologie à propos des «espèces de transition ».

Deux auteurs vont donner à la géographie botanique sa véritable dimension : Alexandre de Humboldt et Augustin Pyramus de Candolle. Bien que tous les deux aient reçu une formation de minéralogiste ou de géologue, ils ont une pratique radicalement opposée quant au rapport au «terrain » comme pratique scientifique.

Pour Humboldt les végétaux doivent être étudiés dans leur rapport avec les facteurs physiques (altitude et latitude) mais aussi dans leurs liens avec l'histoire des hommes, et ce grâce au travail de terrain. Humboldt parcourt l'Amérique latine pendant quatre ans, accompagné du botaniste Aimé Bonpland, et rédige en 1805 un Essai sur la géographie des plantes. En revanche Augustin Pyramus de Candolle, dans l'article "Géographie botanique » du Dictionnaire des sciences naturelles de Frédéric Cuvier, publié en 1820, a travaillé surtout à partir des collections, des herbiers, des jardins botaniques, bien que lui-même ait beaucoup voyagé. Comparant les flores de tous les pays issus de la littérature, de Candolle est le premier à se référer à une région méditerranéenne dans son article de 1820 où il divise la terre en une vingtaine de régions botaniques. Mais il avait déjà effectué ce découpage dans la réédition de la Flore française de Lamarck en 1805 accompagnée d'une carte botanique où il distingue cinq régions dont la Méditerranée (Drouin in a: 153). Or cette notion est issue de la lecture qu'a faite de Candolle du travail de son maître et ami René Desfontaines après un voyage en Tunisie et en Algérie (p. 154), ainsi que d'autres travaux rapportés de voyages, en Égypte notamment.

Cette géographie botanique est à l'origine de toute une réflexion de de Candolle sur la distribution des plantes qui inspirera Darwin. Il insiste sur la distinction entre la «station » d'une plante, le milieu physique dans lequel elle vit (la lumière, l'eau) et son «habitation », la région ou le continent où l'on peut la rencontrer. Or les contraintes du milieu qui ne s'exercent pas mécaniquement, ainsi que la compétition entre les plantes (Drouin 1994 : 78), ne suffisent pas à expliquer la dispersion des espèces car dans 
des lieux où les conditions du milieu sont identiques, mais situés dans deux continents, on ne trouve jamais exactement les mêmes espèces. Aussi, si les stations sont liées à des causes agissant actuellement, « les habitations pourraient bien avoir été en partie déterminées par des causes géologiques qui n'existent plus aujourd'hui ». C'est ce décalage entre la dispersion virtuelle des plantes et leur habitation actuelle qui nécessite le détour par l'histoire de la terre.

L'expédition de Morée donna à Bory de Saint-Vincent une occasion d'appliquer les préceptes du maître genevois. Mais à l'inverse de ce dernier, il lia le travail de terrain et de laboratoire qui donnera lieu à la publication de la Flore de Morée. Là c'est le travail de terrain - la critique des synonymes, l'indication précise du site d'habitation... - qui permet à Bory de prendre fait et cause pour de Candolle dans la controverse larvée qui l'oppose à Humboldt.

Donc la notion de région méditerranéenne n'a pas été élaborée par les missions scientifiques, lesquelles s'inscrivent au contraire dans un ensemble de collectes effectuées par des botanistes voyageurs.

La Grèce est définie du point de vue zoologique comme «contrée de transition » entre les trois continents : Europe, Asie, Afrique. Pour Brullé, l'entomologie de la Morée et des Cyclades est «mixte» car participant des trois continents. Or pour les deux Geoffroy Saint-Hilaire (Étienne et son fils Isidore) de telles contrées de transition, si elles ne permettent pas d'identifier de nouveautés sur le plan de la faune (mammifères, oiseaux), sont le lieu d'émergence de formes intermédiaires ou «variétés de localité ». Ces « demi espèces » permettent d'étudier la formation des espèces ou la transition de l'une à l'autre (Krimbas in b : 164). Ainsi l'étude in situ des variétés de mammifères ou «espèces affines » est la condition de l'adoption d'un programme évolutionniste opposé à celui de Cuvier.

\section{Beaux-arts : l'évaluation de l'art des peuples d'où les valences contradictoires du "primitif "}

Dans ce domaine également on retrouve les tensions évoquées plus haut: changement du régime d'historicité et valeurs respectives du «terrain » et des écrits des Anciens.

L'évaluation de l'art à l'intérieur duquel a été pris le mot «primitif» nous servira de guide pour élucider le rapport au temps. Elle a connu plusieurs acceptions au cours de deux parallèles effectués à propos de l'art grec, tout d'abord avec l'art égyptien, puis avec l'art mycénien. Trois sortes d'échelle ont tour à tour servi à l'exercice. L'échelle de l'origine : l'art d'un peuple a d'abord été évalué en fonction de son antériorité dans l'ordre de l'invention déclinant ce qui suit comme imitation. Ou bien il est affecté à un degré sur une échelle de perfection ou un vecteur de développement qui va de zéro au sublime. Enfin l'échelle chronologique, pour nous la plus 
proche, n'apparaît que tardivement car elle suppose l'éviction des précédentes. Ce sont les deux premières échelles qui vont être appliquées au parallèle entre l'art grec et l'art égyptien. La troisième est liée à l'acception de primitif qui nous est familière; elle émergea avec la confrontation de l'art grec et de l'art mycénien.

S'entremêle avec ces parallèles la controverse sur les nécessités du voyage par rapport à l'homme de cabinet qui opère des reconstructions de papier.

Égypte et Grèce : modèle grec (esthétisation des Grecs)/ art égyptien primitif

Appliquée au parallèle Grecs/Égyptiens, l'évaluation selon l'ordre de l'invention (Petridou in b : 259) ou bien sur une échelle de perfection offre trois possibilités.

1) Soit les Grecs sont à la fois les inventeurs et ceux qui ont amené l'art au plus haut degré de perfection, comme en témoigne la "simplicité de l'art grec » d'après Winckelmann. Car selon ce dernier - dans l'Histoire de l'art chez les Anciens (1764, traduite en français en 1789) - les édifices de l'Antiquité, les monuments, offrent une idée de la vie publique et privée des Anciens. L'histoire de l'architecture d'un peuple éclaire l'histoire de sa civilisation.

Oulebsir (in b) analyse le retour à la Grèce antique amorcé dès le milieu du XVIII ${ }^{\text {e }}$ siècle par le peintre James Stuart et l'architecte Nicholas Revett envoyés en Grèce par la Société des Dilettanti entre 1751 et 1753. L'objectif des Topographical Tour était de constituer des modèles de dessins architecturaux utiles aux contemporains.

2) Soit les Égyptiens sont à la fois les inventeurs et ceux qui ont porté au dernier degré de perfection l'art de l'architecture. C'est l'option de Vivant Denon qui publie en 1802 son Voyage dans la Basse et la Haute Égypte pendant les campagnes du Général Bonaparte. C'est aussi celle des membres de l'expédition d'Égypte. Entre 1809 et 1822 sont publiés les cinq tomes des planches des Antiquités de la Description de l'Égypte (sous la direction d'E. Jomard) 1809-1828.

3) Soit les Égyptiens «ont eu dans les arts le mérite de l'invention, mais n'ont pas su les conduire à ce degré de perfection où les portèrent les Grecs » comme le dit Quatremère de Quincy qui fut secrétaire perpétuel de l'Académie des Beaux-Arts de 1816 à 1839 à Paris (ibid. : 294). Pour Quatremère, qui a une conception cyclique du temps correspondant aux âges de la vie, les éléments positifs de l'art égyptien n'ont pas été développés et en sont restés à l'âge de l'enfance.

Son parallèle entre art grec et art égyptien procède de sa doctrine de l'imitation. Quatremère veut montrer que l'imitation de la nature ne procède 
pas entre deux termes, l'art et la nature, mais entre trois. Le véritable imitateur ne part pas de la nature mais la réduit en système. Il part du type idéal construit à partir de la généralisation des lois et des raisons qui relient la nature et les œuvres d'art: action du climat, des facultés morales, de la religion... Distinguant ensuite l'adoption, c'est-à-dire «l'acte de recevoir dans leur entier le système, les règles et le goût d'une architecture » et l'emprunt d'éléments d'une autre architecture, il déclare que le rapport entre les deux architectures, grecque et égyptienne, n'est fondé que sur l'emprunt.

C'est grâce au patronage de Quatremère qu'Abel Blouet est nommé directeur de la section d'architecture et de sculpture de l'expédition de Morée (ibid.). Ce dernier partage les vues de son maître pour qui l'histoire de l'architecture doit faire connaître quel est le plus parfait des divers systèmes de l'architecture qu'on rencontre dans le monde. Aussi le Jupiter Olympie de Quatremère, publié en 1814, est le point de départ des fouilles d'Olympie d'Abel Blouet. Néanmoins la pratique des fouilles va modifier implicitement les positions de Blouet (ibid. : 299).

Le passé de la Grèce étant l'objet d'une réappropriation symbolique, cela nécessite la mise au point de procédés de restauration ou de reconstruction sur papier qui rétablissent les parties manquantes des monuments en ruine du Péloponnèse. Là comme en géographie les trois sources ou principes de reconstruction sont loin d'accorder le primat à l'observation directe. 1) Les écrits des Anciens - le Périegèse de Pausanias - forment la première source. Quatremère utilise les descriptions des écrivains grecs et romains ainsi que celles des voyageurs pour recréer les monuments du passé car «là où la ruine ne suffit pas ou manque, c'est le texte ancien qui supplée » (p. 256).

2) La comparaison entre le temple de Jupiter à Olympie et d'autres temples comme le Parthénon est utilisée par Quatremère (ibid. : 312 ; voir également Tournikiotis in b : 326). Grâce à la technique du dessin qui «constitue le trait d'union entre l'architecture et les mathématiques », Quatremère superpose le calque des deux temples, ceux d'Olympie et d'Athènes, ce qui autorise la « restitution fidèle » d'un temple octastyle.

3) À partir des vestiges découverts au cours des fouilles, Blouet propose une restitution du temple hexastyle. Mais il le fait aussi en fonction de la hauteur rapportée par Pausanias (Tournikiotis in b : 328) car la réalité visible sur le terrain est corrigée grâce à la bibliographie. Le livre vaut plus que la pierre.

Les monuments primitifs de l'art mycénien (Morée) et l'art grec

En juillet 1829 la décision est prise par les trois puissances (France, Russie et Angleterre) de confier aux troupes françaises le soin de faire évacuer la Morée par les troupes du général Ibrahim. Charles X y adjoint une expédition scientifique où Quinet est recruté par l'Institut comme philologue dans 
la section Archéologie de l'expédition de Morée. Dans La Grèce moderne et ses rapports avec l'Antiquité, ouvrage publié en 1830, il pratique deux types de comparatisme pour décrire Mycènes et Tirynthe. Comparatisme philologique : la porte de Mycènes est comparée à Homère, Tirynthe aux vers d'Hésiode, mais aussi comparaison formelle de ces «monuments primitifs » avec les monuments égyptiens. Les autres voyageurs les qualifiaient de «monuments étranges et primitifs» (Polychronopoulou in b : 289).

Surtout, appliquant les idées de Schelling qui développait le sentiment d'une harmonie intime de la nature et de l'histoire, Quinet inverse le sens de l'imitation: ce sont les pentes découvertes des montagnes qui imitent les constructions cyclopéennes...

\section{La mise au temps des Anciens, dans une antiquité au présent}

L'évaluation des peuples sur une échelle de perfection couplé avec le déterminisme géologique autorise ces télescopages des périodes historiques qui nous étonnent tant aujourd'hui où l'on est rentré dans un « régime d'historicité » qui met l'indigène complètement «hors du temps ». Récurrentes en effet à l'époque de nos expéditions sont les comparaisons entre les Bédouins et les patriarches bibliques, entre les Francs médiévaux et l'époque homérique, entre l'occupation romaine et l'impérialisme français.

La première image qu'on avait du Bédouin était celle du pillard issu de l'imaginaire des pèlerins médiévaux pour qui les nomades sont quasiment des démons. Volney inaugure la comparaison avec les patriarches de l'Ancien Testament (Moussa in a). Le renversement et l'image romantique apparaissent lors de l'expédition d'Égypte avec Coutelle. Le goût du pillage vient de l'esprit de liberté du désert. De même qu'ils se déplacent en marge des espaces habités, parcourant indéfiniment une étendue stérile qui constitue un non-lieu, les Bédouins semblent vivre hors du temps, répétant les rituels des premiers âges (ibid. : 212). La promotion par l'Antiquité passe non seulement par la comparaison de l'hospitalité avec les patriarches mais aussi avec l'Antiquité grecque, les banquets de l'Odyssée.

Yakovaki (in b) analyse le fameux passage de Bory sur la montagne d'Hyères. S'opère une triple «confusion » d'images, superposition entre le relief de la Provence et celle du Péloponnèse, entre relief et ruines, et entre les traces de nos « seigneurs croisés ou des Vénitiens » et les acropoles de la Grèce. Cette dernière comparaison vient d'un livre emporté par Bory, la Chronique de Morée, texte qui remonte à l'histoire de la principauté du Péloponnèse, établie par les chevaliers francs après la première croisade, et publié en 1825.

Bory comme Puillon de Boblaye veulent reconnaître la présence française sur le sol du Péloponnèse en mettant côte à côte ruines médiévales et ruines de l'Antiquité (p. 206). Pour Bory les Français sont des libérateurs, à l'époque de Napoléon ainsi qu'au Moyen Âge. 


\section{L'Algérie: de la Rome antique à la Grèce}

Dès le débarquement en 1830 les militaires manifestent un intérêt spontané pour l'archéologie, marqué en particulier dans les carnets de marche des unités (p. 180), ce qui amène le ministère de la Guerre à souhaiter que soit opéré un bilan confié à l'Académie des inscriptions et belles lettres. L'unité de la Méditerranée est envisagée en fonction de la succession des dominations carthaginoise puis romaine, vandale, arabe (Dondin-Payre in $\mathrm{b}: 183$ ). Les termes de la comparaison ne sont intelligibles que s'ils sont replacés dans un espace doublement polarisé intégrant également l'incomparable. Une équation entre deux termes signifie l'opposition de ces termes à un troisième.

Les premiers témoignages archéologiques - les mégalithes trouvés par le capitaine Rozet entre Alger et Oran en 1831 - sont immédiatement rapprochés des «tombeaux druidiques ou celtiques». À la fin du siècle ils seront comparés à ceux élevés par nos «aïeux les Gaulois ».

L'intérêt pour les Antiquités romaines naît dès le début (Frémeaux 1984). Car il y a un parallèle entre la colonisation et l'administration romaine et française reposant sur l'équation: " population européenne = romaine, opposée aux indigènes » (Romains $=$ Français ; grottes ou maisons de chaumes des Gaulois = gourbis arabes, ou germains).

En Algérie les deux principaux membres dans la section Beaux-Arts étaient Ravoisié, un ancien élève des Beaux-Arts de Paris, et Delamare, capitaine d'artillerie et dessinateur à ses moments de loisir (Oulebsir in a : 250). Enfin la carte du colonel Carbuccia témoigne de cette « romanisation » de l'Algérie qui influença tout le XIX ${ }^{\mathrm{e}}$ siècle.

Le choix d'Amable Ravoisié provient du fait qu'à 28 ans il avait participé à l'exploration scientifique de Morée en 1829-1830 où il avait produit, en collaboration avec Abel Blouet, les relevés des monuments de la Grèce (Oulebsir in b : 301). Comme la carte pour le géographe, le dessin d'architecture est la technique privilégiée par Ravoisié qui rassemble 700 dessins de monuments romains : vue d'ensemble ou perspective - plans, coupes et élévations et vues - ainsi que des « restitutions primitives » ou restaurations de l'édifice (Oulebsir in a : 252, 268).

La vue générale de Djemila est représentative de la formation académique de Ravoisié et est fondée sur le contraste entre différents plans. Le premier niveau offre une vue des bases de colonnes d'un temple; le second une vue plus lointaine : les colonnes des propylées, l'arc de triomphe; enfin, dans la partie droite du troisième niveau, le camp militaire français. L'opposition temporelle des ruines du passé et du présent s'atténue si l'on considère le camp militaire inscrit à l'intérieur du paysage et à l'échelle des vestiges antiques, comme pour vouloir confondre le passé avec le présent (ibid.: 266). Au-delà de l'appropriation symbolique, le dessin prépare le déplacement du monument. Ravoisié élabora une restauration complète de l'arc de 
triomphe de Djemila en vue d'un projet de transfert à Paris qui ne vit pas le jour.

Le capitaine Delamare, qui participe à la conquête de l'Algérie, effectue de nombreux dessins archéologiques et collectionne les antiquités. Ces dessins ne comportent pas de personnages ou de végétation: si l'on constate la présence de quelques indigènes, il y a en revanche absence presque totale des Européens alors que l'on sait que Delamarre était accompagné de soldats pendant ses expéditions (Dondin-Payre in a : 233). L'attention que Delamarre porte aux vestiges de l'Antiquité témoigne de la fascination de l'armée pour l'occupation romaine de l'Algérie. Elle y voyait un exemple à suivre, comme une justification à la réintroduction de la civilisation, la période arabe et turque n'étant que des parenthèses (p. 236).

La carte dressée par le colonel Carbuccia en pleines opérations militaires qui s'étalèrent de 1848 à 1850 donne un troisième témoignage de cette romanisation de l'Algérie (Colonna in a). Les traces des Romains - routes ou forteresses - sont si nombreuses qu'on a l'impression que « le Maghreb met devant nos yeux les sociétés de l'Antiquité figées dans un éternel présent », impression que véhicule Masqueray dans son grand livre, la Formation des cités publié en 1885 (ibid. : 57).

Modèle fustelien et hellénisation diffuse de l'Algérie au $x^{e}$ siècle

En comparant les régions berbères de l'Algérie, Kabylie, Aurès et Mzab, en y discernant une organisation fondée sur une assemblée et une coutume codifiée, Masqueray, dans sa thèse, crée le modèle de la cité berbère, modèle de portée presque universelle (Colonna in $\mathrm{b}:$ 209-225).

Après le détournement opéré par Durkheim en 1893 de l'œuvre de Masqueray on aurait pu penser à un oubli radical du modèle de Fustel de Coulanges. Or, selon Fanny Colonna, on assiste à une latence du modèle fustelien en littérature, durant la première moitié du $\mathrm{xx}^{\mathrm{e}}$ siècle (École des algérianistes entre 1900 et 1950 et École nord-africaine des lettres de Camus et Audisio entre 1935 et 1950), mais également dans le discours indigéniste ou en ethnologie.

À partir d'une thématique à trois volets - la présence de ruines de grande civilisation, leur accord avec le paysage, et la présence d'une race humaine éternellement jeune - celle-ci permet d'exploiter une des virtualités du modèle fustelien, l'unification de Rome et de la Grèce, autorisant le glissement vers l'hellénisation qui a un triple effet.

D'une part cette référence à un peuple éternellement jeune par Camus néantise l'islam. Les élèves d'Evans-Pritchard des années 1960 - PittRivers et Peristiany (1963 et 1966) - vont naturaliser la Méditerranée en effaçant l'islam. D'autre part la Grèce représente une sorte d'universel archaïque renvoyant aux auteurs du XIX ${ }^{\mathrm{e}}$ siècle. L'helléniste Louis Gernet, dont la carrière se déroule à Alger de 1921 à 1948, joue un rôle essentiel. 
C'est Gernet, ami de Herz, Mauss et Granet, qui orientera Berque vers la sociologie durkheimienne et le Maroc après un diplôme de grec.

Enfin l'archaïque grec suppose, par un curieux retournement, l'effacement de Rome et de la christianisation que Colonna réfère à l'anticléricalisme de l'entreprise coloniale. D'où la paganisation de l'Antiquité pratiquée par Mouloud Mammeri et Mouloud Feraoun (ibid. : 220).

Ces trois expéditions conjuguant la science et la conquête militaire, auraient pu conduire à la dénonciation pure et simple de la connaissance qui en résulta. Or M.-N. Bourguet (a : 15), à l'inverse des subaltern studies, oppose Foucault à Saïd et invite à considérer les expéditions comme des dispositifs, à la fois opération d'intervention et expérience de connaissance qui fabriquent, d'un même mouvement, du savoir et du pouvoir.

$\mathrm{Si}$ on inscrit les modalités d'observations et les pratiques discursives de ces expéditions dans une temporalité plus vaste, force est de constater la complexité des rapports qui s'instaurent entre le terrain, géologique ou archéologique, la carte et le texte (ceux des Anciens comme les récits de voyages). Le processus de territorialisation de la connaissance amorcée dans la seconde moitié du XVIII ${ }^{e}$ siècle n'est donc pas univoque comme en témoigne le retour du paradigme philologique au siècle suivant. À l'inverse, dans le domaine des beaux-arts, les différentes échelles d'évaluation concurrentes n'ont pas encore fait place à l'évolution unilinéaire, tout en ayant postulé une relation forte entre une population et la terre qu'elle occupe.

Or, en cette fin de siècle, par un double retournement, l'anachronisme retrouve le droit de citer tandis que certains courants de l'anthropologie anglo-saxonne déterritorialisent la culture.

IRD/EHESS. 


\section{BIBLIOGRAPHIE}

AMSElle, J.-L.

1996 Vers un multiculturalisme français. L'empire de la coutume, Paris, Aubier.

Berque, J.

1956 «Cent vingt-cinq ans de sociologie maghrébine», Annales ESC, 11 (3): 296-324.

DROUIN, J.-M.

1994 «Ludus vitalis», Rivisita de Filosofia de las Ciencias de la Vida, II (3): 69-89.

FABIAN, J.

1983 Times and the Other. How Anthropology Makes it Objects, New York, Columbia University Press.

FrÉMEAUX, J.

1984 «Souvenirs de Rome et présence française au Maghreb : essais d'investigations », in J.-C. Vatin (dir.), Connaissances du Maghreb. Sciences sociales et colonisation, Paris, Éditions du CNRS : 29-46.

1996 «Pertinence et fonction de la frontière Tell-Sahara 1830-1960», in Mélanges Charles-Robert Ageron, Études réunies et préfacées par Bdeljelil TEMIMI, Publications de la Fondation Temimi pour la recherche scientifique et l'information (FTERSI), t. I : 251-267.

HARTOG, F.

1995 «Temps et histoire "Comment écrire l'histoire de France" », Annales SS, nov.-déc. (6) : 1219-1236.

Nordman, D.

1996 Profils du Maghreb. Frontières, figures et territoires (XVIII ${ }^{e}-X X^{e}$ siècle), Rabat, Université Mohammed V, Publication de la Faculté des lettres et sciences humaines («Essais et études»18).

\section{RÉSUMÉ}

Les deux ouvrages collectifs analysés dans cette chronique sont consacrés à la construction de l'espace méditerranéen à partir des expéditions scientifiques et militaires en Égypte, en Morée et en Algérie (fin XVIII ${ }^{\mathrm{e}}$-première moitié du XIX ${ }^{\mathrm{e}}$ siècle). Le modèle de ces expéditions fut transféré au sud du Sahara, en particulier en Sénégambie à l'époque de Faidherbe. La perspective adoptée souligne la complexité des rapports entre science et empire, et la construction des pratiques mises en œuvre conduit à un vaste mouvement de "territorialisation du savoir », socle sur lequel s'édifieront les sciences sociales de la fin du Xix ${ }^{\mathrm{e}}$ siècle. 


\section{ABSTRACT}

Territorializing Knowledge and Inventions in the Mediterranean Basin. - The two edited books under review focus on how a "Mediterranean space" was constructed out of the scientific and military expeditions, from the late 18th to the mid-19th century, in Egypt, Morée and Algeria. This expedition model was transferred south of the Sahara, in particular to Senegambia at the time of Faidherbe. Emphasis is laid on the complexity of relations between science and empire. "Constructing the practices" that were put to use led to a vast trend to "territorialize knowledge", which served as the grounds for edifying the social sciences at the end of the 19th century.

Mots-clés/Keywords : Méditerranée, antiquité, cartographie, géographie, histoire des sciences, primitivisme, territorialisation/Mediterranean Basin, ancient times, cartography, geography, history of science, primitivism, territorialization. 